\title{
On Quasimetrizability of Quasicone Metric Spaces
}

\author{
M. Aphane and S. P. Moshokoa \\ Department of Mathematics and Statistics, Tshwane University of Technology, Pretoria 0008, South Africa
}

Correspondence should be addressed to S. P. Moshokoa; moshokoasp@tut.ac.za

Received 11 May 2015; Accepted 23 June 2015

Academic Editor: Salvatore A. Marano

Copyright (C) 2015 M. Aphane and S. P. Moshokoa. This is an open access article distributed under the Creative Commons Attribution License, which permits unrestricted use, distribution, and reproduction in any medium, provided the original work is properly cited.

\begin{abstract}
The aim of this work is to extend interesting results on the metrizability of cone metric spaces as it appears in the literature. In this paper we appeal to quasiuniformities and uniformities to prove that a quasicone metric space is qausimetrizable, and from our results we will deduce that every cone metric space is metrizable; our approach is more on bitopological and topological properties and differs from the one used by the papers mentioned above but affirms some of their results.
\end{abstract}

\section{Introduction}

Ordered spaces and cones have a wide application in various branches of mathematics, for example, in applied mathematics, optimization theory, and computer science. There are many generalisations of metric spaces in the literature; for instance, we have pseudometric spaces and quasimetric spaces. One also obtains such a generalisation by replacing the codomain of a metric space $[0, \infty)$ when equipped with the usual metric with an ordered Banach space. Then one obtains a generalization of a metric space, referred to as a cone metric space; we refer to the paper by Long-Guang and Xian [1]. Since then many fixed points results in metric spaces were extended to the class of cone metric spaces; see [1], for example.

Furthermore, it is shown in the literature that cone metric spaces can be regarded as metric spaces topologically [2, 3] but that the study of fixed point results may still be worthwhile, since not all fixed point results in cone metric spaces can be reduced to similar results in metric spaces. The initial papers on cone metric spaces and quasicone metric spaces focused on fixed point results and recently it has become an interesting problem to find topological relationships between metric spaces and cone metric spaces. More specifically, the metrizability problem for cone metric spaces has received serious attention [2-5]. The purpose of the paper is to study bitopological properties of quasicone metric spaces, and we will show that a quasicone metric space can be regarded as a quasimetric space in the bitopological setup. However, it does not mean that all the results in quasicone metric spaces, especially those on fixed point theory, can be reduced to similar results in quasimetric spaces. We employ similar constructions as in the cone metric space but, more importantly, we also apply a totally new approach via quasiuniformities to explicitly construct a quasimetric space from a quasicone metric space. Since cone metric spaces are quasicone metric spaces and, not conversely, our results generalize and extend those in the literature. We shall show that all such constructions yield the same bitopological space; we finally discuss similar results in the setting of cone metric spaces and metric spaces.

\section{Preliminaries}

Let $X$ be a nonempty set; a function $d: X \times X \rightarrow[0, \infty)$ satisfying, for all $x, y, z \in X$,

$$
\begin{aligned}
\text { (i) } d(x, x) & =0 \\
\text { (ii) } d(x, y) & \leq d(x, z)+d(z, y)
\end{aligned}
$$

is called a quasipseudometric on $X$ and the pair $(X, d)$ is called a quasipseudometric space. If in addition to (i) and (ii) the function $d$ satisfies (iii) $d(x, y)=d(y, x)$ then $d$ is called pseudometric and the pair $(X, d)$ is referred to as a pseudometric space. It is well known that a pseudometric on $X$ is a metric if $d(x, y)=0$ is equivalent to $x=y$. Now 
quasipseudometric that satisfies $(\mathrm{i})^{\prime} d(x, y)=0$ if and only if $d(x, y)=0$ is referred to as quasimetric on $X$. Clearly we see that every metric on $X$ is quasimetric on $X$ but not conversely. When $d$ is a quasimetric (metric) on $X$, we shall refer to the pair $(X, d)$ as a quasimetric space (metric space).

Let $X$ be a nonempty set and let $\mathcal{U}$ be a filter on $X \times X$. We denote by $\Delta=\{(x, x): x \in X\}$. Suppose that the following hold for $X$ and $\mathcal{U}$ on $X$ :

(a) $\Delta \in U$, for every $U \in \mathcal{U}$.

(b) For every $U \in \mathcal{U}$ there exists $V \in \mathcal{U}$ such that $V \circ V \subseteq$ $U$.

Then the filter $\mathcal{U}$ is called a quasiuniformity on $X$, and the pair $(X, \mathcal{U})$ is referred to as a quasiuniform space. Note that a quasiuniformity $\mathcal{U}$ is a uniformity on $X$ if and only if, for every $U \in \mathcal{U}$, the inverse of $U$, denoted by $U^{-1}$, contains a member of $\mathcal{U}$. So every uniformity on $X$ is a quasiuniformity, but the converse is not necessarily true.

It is well known [6] that given a quasiuniform space $(X, \mathcal{U})$, we get a bitopological space. The bitopological space is obtained in the following manner. A subset $A$ (which is open) belongs to the topology induced by $\mathcal{U}$, if for every $a \in A$ there exists $U \in \mathcal{U}$ such that $U[a] \subset A$, where $U[a]=\{x \in X:(a, x) \in U\}$. Let $\mathcal{U}^{-1}=\left\{U^{-1}: U \in \mathscr{U}\right\}$; then $\mathscr{U}^{-1}$ is a quasiuniformity on $X$, called the conjugate of $\mathcal{U}$. Let the topology on $X$ induced by $\mathcal{U}$ be denoted by $\tau_{\mathcal{U}}$; then associated with a quasiuniform space $(X, \mathcal{U})$ we obtain the bitopological space $\left(X, \tau_{\mathscr{\ell}}, \tau_{\mathcal{U}^{-1}}\right)$.

\section{Main Results}

The following definition is given in [4].

Definition 1. Let $E$ be an ordered vector space. An element $e \in$ $E$ is called an order unit if for each $x \in E$ there exists a real number $\lambda$ such that $x \leq \lambda e$.

Definition 2 (see [4]). Let $X$ be a nonempty set and $E$ an ordered Banach space with a cone $K$ in $E$ such that $\operatorname{int}(K) \neq \emptyset$ and $K$ is closed. A function $d: X \times X \rightarrow E$ is a cone metric if

(i) $d(x, y)=0$ if and only if $x=y$;

(ii) $d(x, y)=d(y, x)$ for all $x, y \in X$;

(iii) $d(x, y) \leq d(x, z)+d(z, y)$ for all $x, y, z \in X$.

In this case $(X, E, K, d)$ is called a cone metric space.

Example 3. Let $X$ be the set of real numbers, and $E=X \times X$. Put

$$
K=\{(x, y) \in E: x, y \geq 0\} .
$$

For $\alpha \geq 0$, we define

$$
d: X \times X \longrightarrow E
$$

by

$$
d(x, y)=(|x-y|, \alpha|x-y|) .
$$

Then $(X, E, K, d)$ is a cone metric space.
Recently, the notion of a cone metric space has been extended to that of a quasicone metric space. Actually one such notion is found in [7] and later generalised by Shadda and Md Noorani in [8]. We recall the following.

Definition 4 (see [8]). Let $X$ be a nonempty set and let $E$ be an ordered vector space with cone $K$. The function $d: X \times X \rightarrow$ $E$ is called a quasicone metric if

(i) $0 \leq d(x, y)$ for all $x, y \in X$;

(ii) $d(x, y)=0=d(y, x)$ if and only if $x=y$;

(iii) $d(x, y) \leq d(x, z)+d(z, y)$ for all $x, y, z \in X$.

In this case $(X, E, K, d)$ is called a quasicone metric space.

Example 5. Let $X$ be the set of real numbers, and $E=X \times X$. Put

$$
K=\{(x, y) \in E: x, y \geq 0\} .
$$

For $\alpha \geq 0$, we define

$$
d: X \times X \longrightarrow E
$$

by $d(x, y)=((x-y), \alpha(x-y))$ when $x \geq y$ and $d(x, y)=$ $(0,0)$, otherwise. Then $(X, E, K, d)$ is a quasicone metric space.

Certainly a cone metric space is a quasicone metric space but not conversely.

We start by partially extending Theorem 1.4 from [4], the proof is straight forward and therefore it is omitted.

Theorem 6. Let $X$ be a nonempty set, let $E$ be an ordered vector space with cone $K$, and let $e \in \operatorname{int}(K)$. For a function $d: X \times X \rightarrow K$, define $\bar{d}: X \times X \rightarrow[0, \infty)$ by

$$
\bar{d}(x, y)=\inf \{\lambda: d(x, y) \leq \lambda e\} .
$$

(i) If $d$ is a quasicone metric, then $\bar{d}$ is quasimetric.

(ii) If $d$ is a cone metric, then $\bar{d}$ is a metric.

Later on in the paper given a quasicone metric space $(X, E, K, d)$ by $\bar{d}$ we denote the quasimetric on $X$ obtained in Theorem 6 . For a vector space $E$ and $a, b \in E$, we define $a \vee b=\left(a_{1} \vee b_{1}, a_{2} \vee b_{2}, \ldots\right)$, where $a=\left(a_{1}, a_{2}, \ldots\right)$ and $b=$ $\left(b_{1}, b_{2}, \ldots\right)$. Now given a quasicone metric space $(X, E, K, d)$, define a function $d^{-1}: X \times X \rightarrow E$ by $d^{-1}(x, y)=d(y, x)$, for all $x, y \in X$. Then $\left(X, E, K, d^{-1}\right)$ is a quasicone metric space. We shall refer to $d^{-1}$ as a conjugate of $d$. Further, let the function $d^{s}: X \times X \rightarrow E$ be defined by $d^{s}(x, y)=$ $d(x, y) \vee d^{-1}(x, y)$, for all $x, y \in X$. Then $\left(X, E, K, d^{s}\right)$ is a cone metric space. Given a cone metric space $(X, E, K, d)$, we note that $d=d^{-1}=d^{s}$.

Let us revisit Example 5.

Example 7. Let $X$ be the set of real numbers, and $E=X \times X$. Put

$$
K=\{(x, y) \in E: x, y \geq 0\}
$$


For $\alpha \geq 0$, we define

$$
d: X \times X \longrightarrow E
$$

by $d(x, y)=((x-y), \alpha(x-y))$ when $x \geq y$ and $d(x, y)=$ $(0,0)$, otherwise. Then $(X, E, K, d)$ is a quasicone metric space. Note that $d^{s}: X \times X \rightarrow E$ is defined by

$$
d^{s}(x, y)=(|x-y|, \alpha|x-y|)
$$

and certainly $\left(X, E, K, d^{s}\right)$ is a cone metric space.

We will write $0 \ll e$ whenever $e \in \operatorname{int}(K)$. Let $(X, E, K, d)$ be a quasicone metric space. For $x \in X$, let $B_{\ll}(x, r)=\{y \in$ $X: d(x, y) \ll r\}$.

Proposition 8. Let $(X, E, K, d)$ be a quasicone metric space. Then the family $\left\{B_{\ll}(x, r): x \in X, 0 \ll r\right\}$ forms a basis for the topology on $X$.

Given a quasicone metric space $(X, E, K, d)$, let us denote the topology induced by $d$ with $\tau_{d}$; then the following is immediate.

Theorem 9. Let $(X, E, K, d)$ be a quasicone metric space; then we get a bitopological space $\left(X, \tau_{d}, \tau_{d^{-1}}\right)$.

From Theorems 6 and 9, we easily deduce that a quasicone metric space is quasimetrizable.

Given a quasicone metric space $(X, E, K, d)$ and $e \in$ $\operatorname{int}(K)$, define $D: X \times X \rightarrow[0, \infty)$ by

$$
\begin{aligned}
& D(x, y) \\
& \quad=\inf \left\{\sum_{i=1}^{n-1} \Lambda\left(x_{i}, x_{i+1}\right): x_{1}=x, \ldots, x_{n}=y\right\},
\end{aligned}
$$

where $\Lambda(x, y)=\left\{d^{\min \left\{k: d(x, y) \ll(1 / c)^{k} \cdot e\right\}}\right\}$ if $d(x, y) \neq 0$, or $\Lambda(x, y)=0$ if $d(x, y)=0$ and $c<1$ is a real number. Note that $D$ is quasimetric on $X$ whenever $d$ is a quasicone metric on $X$; moreover, $D$ is a metric on $X$ whenever $d$ is a cone metric on $X$.

The following is an extension of Theorem 3.4 in [5].

Theorem 10. Let $(X, E, K, d)$ be a quasicone metric space and $e \in \operatorname{int}(K)$ and a positive real number $c<1$. Then there exists a quasimetric $D: X \times X \rightarrow[0, \infty)$ which induces the same bitopological space; that is, one has $\left(X, \tau_{d}, \tau_{d^{-1}}\right)=\left(X, \tau_{D}, \tau_{D^{-1}}\right)$, with $\tau_{d}=\tau_{D}$ and $\tau_{d^{-1}}=\tau_{D^{-1}}$.

The reader should note that the quasimetric $\bar{d}$ constructed in Theorem 6 and the quasimetric $D$ constructed in Theorem 10 may differ when nontopological properties of the spaces involved are under consideration. For bitopological properties we have the following.

Theorem 11. Let $(X, E, K, d)$ be a quasicone metric space. Then the bitopological spaces $\left(X, \tau_{d}, \tau_{d^{-1}}\right),\left(X, \tau_{\bar{d}}, \tau_{\bar{d}^{-1}}\right)$, and $\left(X, \tau_{D}, \tau_{D^{-1}}\right)$ are the same.

Next we provide a new result that proves that a quasicone metric space $(X, E, K, d)$ is quasimetrizable; we appeal to quasiuniformities.
The proof of the next result is similar to the proof of Lemma 13, so we omit the proof.

Theorem 12. Let $(X, E, K, d)$ be a quasicone metric space. Then there exists a quasiuniformity $\mathcal{U}$ of $X$ such that $\tau_{\mathcal{U}}=\tau_{d}$.

Actually, we have the following.

Lemma 13. Let $(X, E, K, d)$ be a quasicone metric space and $e \in \operatorname{int}(K)$ be fixed. Then $\left\{U_{d,(1 / n) e}\right\}$ is a countable base for a quasiuniformity $\mathcal{U}$ on $X$.

Proof. Let $x \in X$ and define $U_{d, e / n}[x]=\{y \in X: d(x, y) \ll$ $e / n\}$. We will show that the collection $\left\{U_{d, e / n}\right\}$ is a base for a quasiuniformity on $X$. We first note that, for each $0<e / n<1$, the set $\Delta \in U_{d, e / n}$; also, we have $U_{d, e /(n+1)} \subset U_{d, e / n}$. Let $n$ and $m$ be positive integers such that $2 n<m$. We will show that $U_{d, e / m} \circ U_{d, e / m} \subset U_{d, e / n}$. Let $(x, y) \in U_{d, e / m} \circ U_{d, e / m}$. Find $z \in X$ such that $(x, y) \in U_{d, e / m}$ and $(y, z) \in U_{d, e / m}$. Clearly, $d(x, z) \ll 2 e / m$; hence $d(x, z) \ll e / n$. Since $(x, z) \in U_{d, e / m} \circ$ $U_{d, e / m}$, this shows that $(x, z) \in U_{d, e / n}$. It follows that $\left\{U_{d, e / n}\right\}$ is a countable base for the quasiuniformity $\mathcal{U}$ on $X$.

We recall the following regarding the quasimetrization problem of quasiuniform spaces.

Theorem 14. A quasiuniform space $(X, \mathcal{U})$ is quasimetrizable if and only if the quasiuniform space $\mathcal{U}$ has a countable base.

Note that, from Lemma 13, if we denote the quasiuniformity $\mathcal{U}$, induced by $d$ with $\mathcal{U}_{d}$, then we have $\tau_{d}=\tau_{\varkappa_{d}}$.

We now obtain the following.

Theorem 15. Let $(X, E, K, d)$ be a quasicone metric space and lete $\in \operatorname{int}(K)$ be fixed. Then the bitopological space $\left(X, \tau_{d}, \tau_{d^{-1}}\right)$ is quasimetrizable.

Proof. Let $(X, E, K, d)$ be a quasicone metric space, let $e \in$ $\operatorname{int}(K)$ be fixed, and let $\mathcal{U}_{d}$ be the quasiuniformity induced on $X$ as obtained by Lemma 13 . The quasiuniformity $\mathcal{U}_{d}$ has a countable base; hence it is quasimetrizable by Theorem 14 .

We now construct a quasimetric $d_{U_{d}}$ on $X$ associated with quasiuniformity $\mathcal{U}_{d}$ in Lemma 13 . The family $\left\{U_{d, e / n}\right\}$ in the proof of Lemma 13 is obtained from the quasicone metric space $(X, E, K, d)$, let $U_{d, 0}=X \times X$. Then

$$
U_{d, e /(n+1)} \circ U_{d, e /(n+1)} \circ U_{d, e /(n+1)} \subset U_{d, e / n} ;
$$

for all natural $n$.

We define a function $f: X \times X \rightarrow[0, \infty)$ by $f(x, y)=$ $1 / 2^{n}$ if and only if $(x, y) \in U_{d, e / n}-U_{d, e /(n+1)}$ and $f(x, y)=0$ if and only if $(x, y) \in U_{d, e / n}$ for each $U_{d, e / n}$. Next we define $d_{u_{d}}: X \times X \rightarrow[0, \infty)$ by

$$
d_{u_{d}}(x, y)=\inf \sum\left\{f\left(x_{i}, x_{i+1}\right): i=0,1, \ldots, n\right\}
$$

over all finite sequences $x_{0}, x_{1}, \ldots, x_{n+1}$ such that $x=x_{0}$ and $y=x_{n+1}$. The function $d_{u_{d}}: X \times X \rightarrow[0, \infty)$ is quasimetric on $X$. Finally, $\tau_{d}=\tau_{u_{d}}=\tau_{d_{U_{d}}}$.

Corollary 16. Let $(X, E, K, d)$ be a cone metric space. Then the topological spaces $\left(X, \tau_{d}\right),\left(X, \tau_{\bar{d}}\right)$, and $\left(X, \tau_{D}\right)$ are the same. 
Corollary 17. Let $(X, E, K, d)$ be a cone metric space. Then there exists a uniformity $\mathcal{U}_{d}$ of $X$ with a countable base such that $\tau_{u_{d}}=\tau_{d}$.

The following confirms the already known result about cone metric spaces and the metrization problem for these spaces; see for example [3-5].

Theorem 18. Let $(X, E, K, d)$ be a cone metric space. Then the topological space $\left(X, \tau_{d}\right)$ is metrizable.

\section{Contractions and Fixed Point Theory}

Let $(X, E, K, d)$ be a quasicone metric space. A map $T$ : $(X, E, K, d) \rightarrow(X, E, K, d)$ is a contraction whenever

$$
d(T x, T y) \leq \alpha d(x, y)
$$

for all $x, y \in X$ and $\alpha \in(0,1]$. Note that if $T:(X, E, K, d) \rightarrow$ $(X, E, K, d)$ is a contraction, then

$$
\bar{d}(T x, T y) \leq \alpha \bar{d}(x, y)
$$

holds for all $x, y \in X$ and $\alpha \in(0,1]$, where $\bar{d}$ is as in Theorem 6. Further, the following

$$
D(T x, T y) \leq \alpha D(x, y)
$$

holds for all $x, y \in X$ and $\alpha \in(0,1]$, where $D$ is the quasimetric on $X$ induced by $d$ defined earlier in the paper. So properties of quasicone metric spaces easily transfer to quasimetric spaces as discussed above in this section of the paper, so we can conclude that some fixed point results for contractive mappings in complete quasicone metric spaces $[8,9]$ may be reduced to similar ones in quasimetric spaces.

\section{Conclusion}

In the paper we show that every quasicone metric space can be regarded as a quasiuniform space, and we used this observation to construct a quasimetric space associated with the quasiuniform space, and this yielded the same bitopological space as the one induced by the quasicone metric space. It is instructive to mention that bitopological properties of quasimetric spaces, like pairwise normality and pairwise paracompactness also hold for quasicone metric spaces. We also showed that the quasicone metric space is quasimetrizable, and we explicitly constructed the quasimetric associated with the quasiuniformity. In the literature, for cone metric spaces different methods are employed and applied to construct a metric, such that the topologies induced by the cone metric and the resulting metric are the same. Similar constructions also work for quasicone metrics and quasimetric spaces and the resulting bitopological spaces are comparable; actually they are the same. In the paper we employed quasiuniformities and uniformities, to discuss a similar problem. Finally, we related our work to the ones done for cone metric spaces, and it is shown that our results extend, support, and confirm the work done for cone metric spaces.

\section{Conflict of Interests}

The authors declare they have no competing interests.

\section{Authors' Contribution}

All the authors have contributed equally, read, and approved the submitted paper.

\section{References}

[1] H. Long-Guang and Z. Xian, "Cone metric spaces and fixed point theorems of contractive mappings," Journal of Mathematical Analysis and Applications, vol. 332, no. 2, pp. 1468-1476, 2007.

[2] M. Asadi, B. E. Rhoades, and H. Soleimani, "The equivalence of cone metric spaces and metric spaces," Fixed Point Theory and Applications, vol. 87, 2012.

[3] W.-S. Du, "A note on cone metric fixed point theory and its equivalence," Nonlinear Analysis, vol. 72, no. 5, pp. 2259-2261, 2010.

[4] Z. Ercan, "On the end of the cone metric spaces," Topology and its Applications, vol. 166, pp. 10-14, 2014.

[5] M. Khani and M. Pourmahdian, "On the metrizability of cone metric spaces," Topology and its Applications, vol. 158, no. 2, pp. 190-193, 2011.

[6] P. Fletcher and W. F. Lingren, Quasi-Uniformities, Marcel Dekker, New York, NY, USA, 1982.

[7] T. Abdeljawad and E. Karapinar, "Quasicone metric spaces and generalizations of Caristi Kirk's theorem," Fixed Point Theory and Applications, vol. 2009, Article ID 574387, 9 pages, 2009.

[8] F. Shaddad and M. S. Md Noorani, "Fixed point results in quasicone metric spaces," Abstract and Applied Analysis, vol. 2013, Article ID 303626, 7 pages, 2013.

[9] E. F. Kazeem, C. A. Agyingi, and Y. U. Gaba, "On quasipseudometric type spaces," Chinese Journal of Mathematics, vol. 2014, Article ID 198685, 7 pages, 2014. 


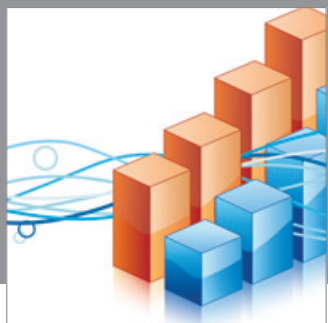

Advances in

Operations Research

mansans

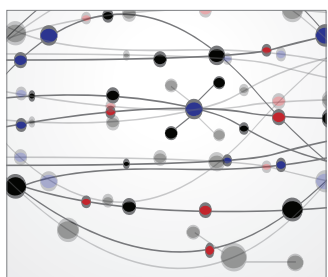

The Scientific World Journal
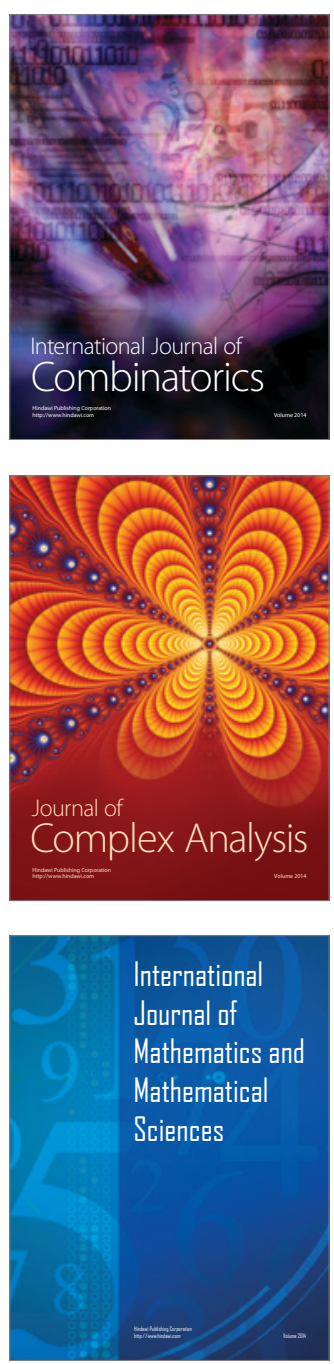
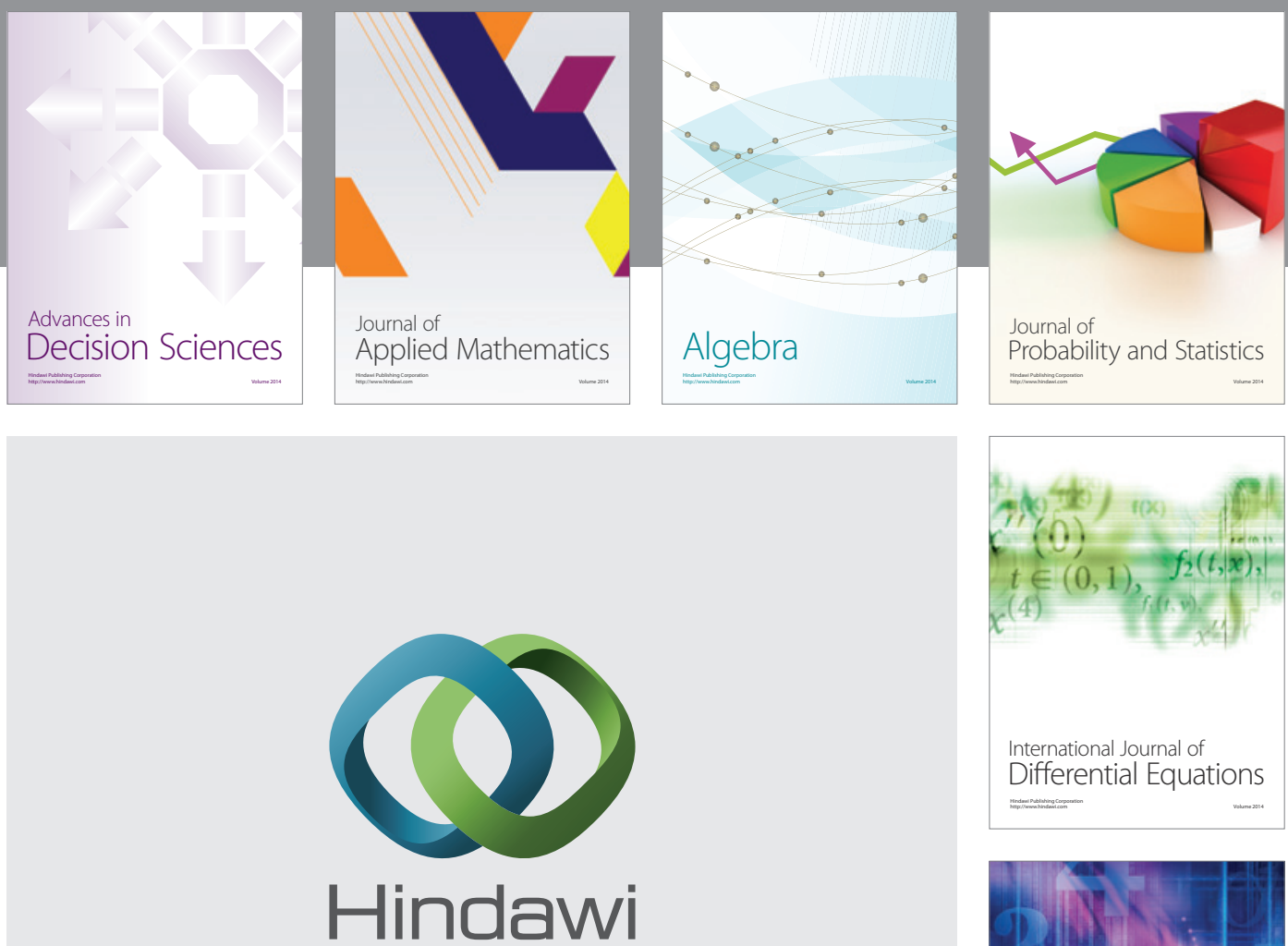

Submit your manuscripts at http://www.hindawi.com
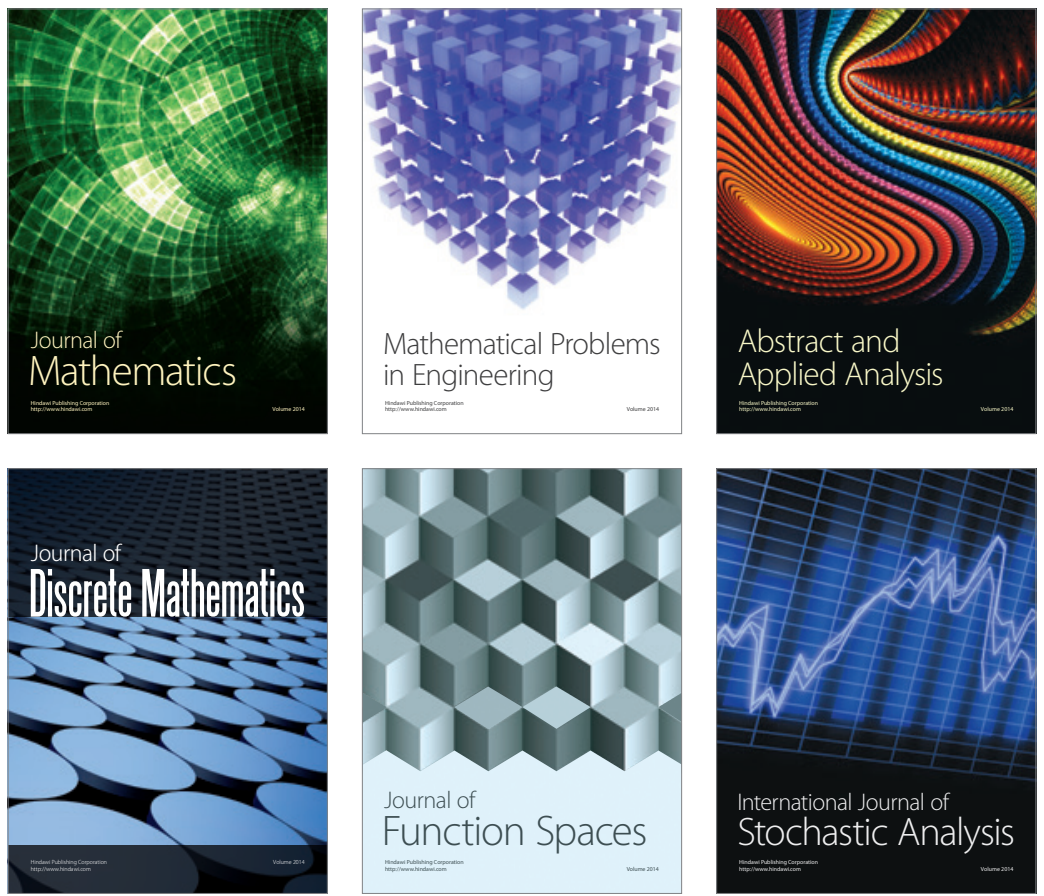

Journal of

Function Spaces

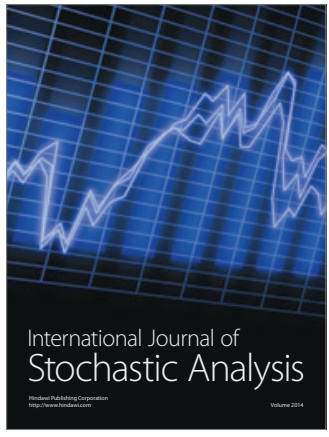

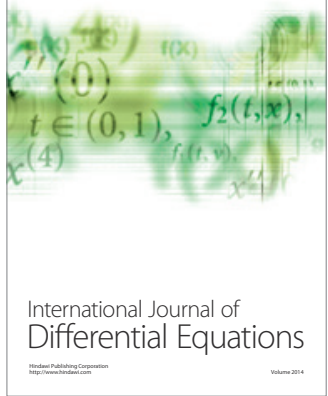
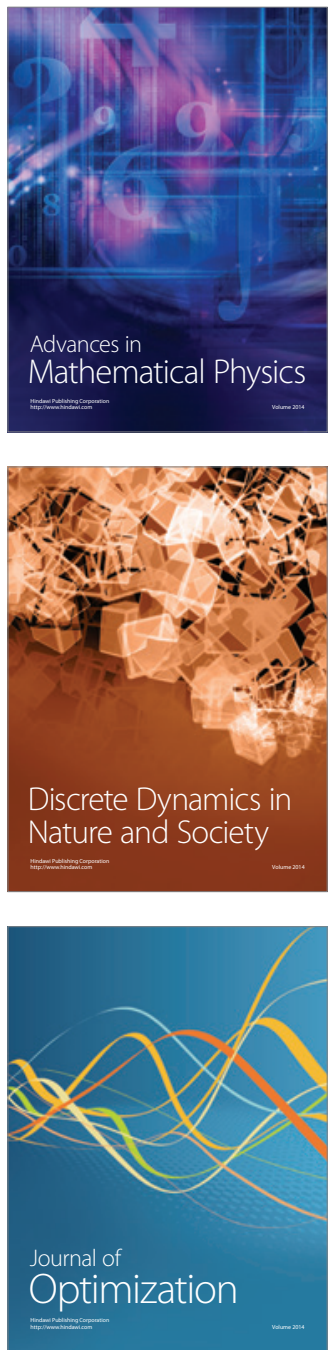